\title{
The influence of hand visualization in tool-based motor-skills training, a longitudinal study
}

\author{
Aylen Ricca* $\quad$ Amine Chellali ${ }^{\dagger} \quad$ Samir Otmane
}

IBISC, Univ Evry, Université Paris-Saclay

\begin{abstract}
Mastering motor skills requires performing the task unconsciously with great speed and accuracy. This is acquired slowly through practice over time. Nonetheless, in domains such as surgery, the training of these skills in the field introduces safety, ethical and economic issues. In this context, immersive VR technologies offer the possibility to recreate real-world situations and allow the trainees to improve their skills in a safe and controlled environment. However, the design of such systems raises new research questions, such as how to represent the user in the virtual environment, and whether this representation can influence motor skills automaticity.

In this work, we focus on how the user's hand representation can impact the training of tool-based motor skills in immersive VR. To investigate this question, we have created a VR simulator for training a tool-based pick and place task, and conducted a user study to evaluate how the user's hand visualization can influence participants' learning performance after a two-week training period. For that purpose, two groups of participants were trained in the VR simulator under one of the two experimental conditions: the presence and the absence of their virtual hands' representation, while a control group received no training. The results of the study show that training on the VR simulator improves the participants' motor task performance when compared with the control group. On the other hand, no difference was observed between the two training groups. This suggests that the user's hand visualization does not always impact tool-based motor tasks training in immersive VR simulators. Indeed, for short-term motor training there was no difference in performance between having a partial embodiment of the user's hands and only the tools representation.
\end{abstract}

Keywords: Avatar; Motor-skills training; Immersive virtual reality.

\section{INTRODUCTION}

A motor skill is the coordination of small muscles in movements usually involving the synchronization of hands and fingers with the eyes [37]. In surgery, motor skills permit to perform several tasks such as instrument handling, tying knots, or suturing. However, even the most basic of these tasks requires several skills such as precision, bimanual dexterity, hand-eye coordination and force control.

To ensure patients' safety during surgical procedures, the execution of those tasks must be performed with higher degrees of efficiency. The mastery of these skills "depends on the ability to perform it unconsciously with speed and accuracy while consciously carrying out other brain functions" [6]. Indeed, motor skills can be performed unconsciously with great speed and accuracy only when reaching the highest level of automaticity, allowing the practitioners to focus their attention on the other factors of the procedure. Master-

*e-mail: aylen.ricca@ibisc.fr

$\dagger$ e-mail: amine.chellali@ibisc.fr

‡e-mail: samir.otmnae@ibisc.fr ing these skills requires repetitive training, usually done on the field under the supervision of more experienced surgeons. However, this method raises several ethical and patient safety issues [36].

Recently, the surgical domain has shown interest in using immersive VR technologies to improve the training of those skills. Immersive VR technologies are interactive systems composed of display and tracking systems coupled to a computer which generate the virtual environment (VE) in which a user can interact [34]. These technologies allow a realistic and interactive simulation of different procedures in a safe and cost-effective environment, while avoiding the ethical and safety issues of traditional in-the-field training methods $[2,3,12]$. In addition, VR simulation allows repeatability, the generation of different tasks and scenarios, and provides objective feedback and evaluation measures [7,8].

Nonetheless, the design of these training systems raises new research questions. For instance, most of immersive VR simulators require the users to wear a head-mounted display (HMD), which allows them to explore and interact with the VE from a first-person perspective but without having direct visualization of their own physical body. Therefore, how the user body parts are represented in the VE and what impact these representations can have on motor skills automaticity remains open questions.

Many surgical tasks require the manipulation of tools to accomplish a manual procedure. In this work, we focus more particularly on how the users' hand representation can influence a tool-based motor task performance, in systems designed to train bimanual dexterity and hand-eye coordination skills.

To investigate this question, we have designed and implemented a VR simulator for a tool-based pick and place task, which was inspired from existing part-task surgical trainers. We have also conducted a user study to evaluate how the users' hand visualization can influence task performance after a two-week training period in the VR simulator.

\section{BACKGROUND}

\subsection{Immersive VR training}

The main motivations of using immersive VR technologies for training are related to situations that are impossible or dangerous to reproduce physically, such as space exploration, firefighters training, or surgical procedures [16]. With the advancements in VR technology, HMDs provide cost-effective solutions for training. In addition, HMDs as training tools have also been shown to be more efficient than non-immersive technologies [40]. When immersed through a HMD, the user can have a first person perspective of the simulated environment thanks to the head tracking. Moreover, when coupled with other devices, it is also possible to track the users' body parts, such as hands and foots, allowing a more natural interaction. One of the problems when wearing a HMD is that the users do not see their own physical bodies. In the context of motor skills training in immersive VR, an open research question is whether visualizing a virtual representation of the self-body improves the training performance. In this work, we focus on motor skills training for surgery, where the users manipulate tools with the hands. Therefore, the self-body representation concerns primarily the users' hands. 


\subsection{The impact of virtual hands characteristics on user experience in VR}

The importance of body-based perception in immersive VR systems has been studied for some time [34,35], showing a high correlation between presence and the degree of association with the virtual body [35] (sense of embodiment). Presence can be defined as "the perceived realness of a mediated or virtual experience" [33], which distinguish between place illusion ("sense of being there") and plausibility illusion ("the illusion that what is apparently happening is really happening") [34]. The sense of embodiment in VR applications refers to the set of sensations arising from "being inside, having, and controlling a virtual body" [23].

Many studies have investigated the influence of hand appearance and control on users' sense of embodiment in different tasks and applicatios [21]. In this context, two characteristics of the virtual hand have been explored; the hand visual fidelity (i.e., visual resemblance between the real and virtual hands) and the hand kinematic fidelity (i.e., replicating the real hand movements on the virtual hands). In general, research has shown that higher levels of kinematic fidelity can influence the users' sense of agency (i.e., sense of having motor control) $[1,30]$ and their whole state of presence [25]. On the other hand, higher levels of visual fidelity have been shown to improve the sense of ownership (i.e., the virtual body is experienced as users' own body) $[1,17,26]$. In addition, hands structural (e.g., extra/fewer fingers) and appearance (e.g., gender models, size) differences can also affect the sense of ownership [22,27,31,32].

In addition to studying how virtual hands characteristics can influence users' experience inside the VE, it is important to determine whether the visualization of the virtual hands can also impact manual tasks performance and motor skills training in VR simulators.

\subsection{Hands visualization in manual tasks and motor skills training}

Caggianese et al. have explored hand-based manipulation tasks in immersive VR [9]. In their experiment, they have compared two different metaphors, handheld controllers vs. mid-air interaction, for block arrangement tasks. Users were able to visualize a virtual hand collocated with their own hand during both conditions. However, the fingers movements were more realistic when the Leap Motion device was used. The results show that controller interactions allowed the participants to carry out the task faster than the hand gestures technique, particularly for the motor tasks involving a combination of several actions and movements (i.e., selection, rotation and positioning). Controllers were also rated as easier to use than mid-air interactions.

For short-term motor skills learning, Ossmy et al. have shown that both visual and kinematic properties of a virtual hand representation affect performance for a task requiring to learn a sequence of finger movements [28]. Indeed, performance increased with bigger virtual hands if motor synchronicity was provided. Motor synchronicity importance was also found to be beneficial for the motor skills learning process involving the upper limp [10]. This suggests that a virtual hand with high kinematic capabilities can improve motor skills learning.

Moreover, Van Nguyen et al. study have shown that adding a static virtual hand during a surgical motor task increases depth perception and gives users additional spatial cues when manipulating tools [39]. Similarly, visual feedback from arms and hands was reported to be better for movement perception in tool-based tasks than the visualization of an isolated tool [18].

On the other hand, other studies have shown that the virtual hands do not have a significant impact on performance for direct and toolbased object manipulation $[25,30]$. Indeed, Batmaz et al. showed that for a pick and place task, tool-based manipulation achieved the same performance as bare hands manipulation in terms of precision for direct and 2D-monitor visualization of the working site [5], and with stereoscopic view [4]. Moreover, Lok et al. concluded that hands visual fidelity of the hand is not required for a block arrangement task through direct manipulation [25]. In addition, their results show that the interaction with physical objects significantly improves participants' performance, due to generating a more realistic interaction. This supports the need of adding physical tools to VR motor skills trainers. Finally, Ricca et al. experiment has shown that having the users' virtual hand visualization does not impact users' task performance during a tool-based motor task [30]. However, it is not clear whether these findings apply for a longer training.

\subsection{Tool-based interactions in VR simulation}

Different approaches are used to design motor skills interactions in VR. For tool-based motor tasks, haptic devices have been frequently used to support these interactions due to the multi-modal feedback which has been shown to be beneficial for skill acquisition. Indeed, force feedback has been recognized as important when performing motor skills, particularly for surgical skills [11, 14, 15, 38], but also for industrial maintenance tasks $[19,29]$. These interactions are particularly interesting for tool-based motor tasks, since a shape-alike tool can be coupled with the device to achieve a better representation and reproduce the same interaction as expected in real procedures $[20,38]$. This suggests that the use of haptic devices is important to support tool-based interactions in VR simulators. Nevertheless, their combination with virtual hands in an immersive VR simulator remains a challenge. While this question is out of this work scope, we have included haptic feedback to support tool-based motor skills training in our simulator as suggested by the literature.

To summarize, although many works have explored users' hand virtual representation and control in VR, their impact on motor skills training remains unclear. Questions on whether having a virtual hand in the VE can impact motor skills training and acquisition remain open, more particularly, for tool-based manual tasks. This work aims to explore the impact of the user's hands visualization on a tool-based motor skill training. The long term object is to improve the design of surgical skills trainers by providing guidelines on the artefacts and characteristics an immersive surgical VR trainer must support to ensure an appropriate training of motor skills.

\section{Methods}

In this work, we investigate whether participants' motor-task performance in VR improves after a two-weeks training period in the simulator, and whether this improvement is influenced by having their hands represented in the VE during training. For this purpose, we have developed a VR simulator, for a bi-manual tool-based pick and place task, and conducted a user study following a pre- postretention-test design. Two training groups and a control group were formed. The first training group was trained with the virtual hands visualization, while the second group was trained without it (tools visualization only). Finally, the control group did not receive training and only participated in the pre-, post-, retention tests.

Our research questions for this study are:

- Would training groups significantly improve their performance in the VR simulators as compared to the control group?

- Would the experimental condition in the VR simulator impact differently the performance of the two training groups?

Our hypotheses were:

- H1) Participants in the training groups would improve their performance (i.e., time, accuracy, and errors) in the VR simulator as compared to the control group.

We hypothesize that the training groups would benefit from the training sessions to automatize the motor task execution, expecting an improvement from the pre-test to the post-test. In addition, we were expecting a retention of the motor skill performance after a short pause, which means that the performance at the post-test would not be significantly different from 
that of the retention-test. On the other hand, although one can expect a small improvement for the control group between the three tests (pre-, post- and retention-test), we were expecting that the training groups improvement would be significantly higher than that of the control group.

- H2) Participants that trained under the virtual hand visualization condition would improve their performance in the VR simulator compared to participants who trained in the no hand condition.

We were expecting that users can improve differently under the two conditions after a longer exposure to the simulator. In particular, we hypothesize that the hand-visualization training condition would more positively affect the users' performance by providing additional spatial and hand-eye coordination cues as suggested by previous research. Therefore, the group trained under the hand-visualization condition is expected to reach a higher performance level in the post-test than the group trained under the no-hand visualization condition.

\subsection{Participants}

Previous studies show that a minimum of 6 participants per group is required for assessing training $[24,41]$. Therefore, a total of 26 participants (16 males, 10 females) were retained for the study $(N=26)$, and were divided into three groups: the control group $(N=9)$ and two training groups $(N=9$ and $N=8$, respectively for training with the virtual hand and the tools only representation). The mean age was $\mu=37.00(\sigma=10.87)$. Nineteen of them were right-handed, six left-handed (23\%), and one ambidextrous. All of them had normal or corrected to normal vision, and twelve wore their correction glasses during the experiment. All of them had previous experience with video games, with thirteen of them being regular players. Thirteen participants reported a previous experience with 3D VEs, and nine participants used haptic devices before this experiment (mainly in demonstrations or previous user studies). Finally, thirteen participants have previously used HMDs.

The institutional ethics committee of Université Paris-Saclay (CER Paris Saclay) approved the experimental protocol beforehand, and all the participants were presented with an informed written consent prior to their participation.

\subsection{Experimental design}

The experiment followed a three-way mixed-design with one between-subjects factor, the group, with three levels: control group (CG), hand-training group (HT) and tool-training group (TT); and two within-subjects factors, the phase, with four levels: the pre-test (PRE), the training (TRAIN), the post-test (POST) and the retentiontest (RET), and two conditions for the VR prototype, the presence of the animated virtual hands $(\mathrm{VH})$ and the absence of them $(\mathrm{NH})$.

All participants $(N=26)$ performed the PRE, POST and RET phases. Each of these phases consisted of a single experimental session (30 minutes mean duration) where participants performed an evaluation test of the pick and place task in the VR simulator under the two experimental conditions. The order of presentation of the conditions was counterbalanced for each group (CG, HT and TT).

The training phase consisted of ten training sessions on the VR prototype performed in two successive weeks (30 minutes mean duration each). Only HT and TT groups participated in the training phase. The HT group was trained under the VH condition while the TT group was trained under the NH condition (see Fig. 1).

\subsection{Experimental task}

The experimental task is a simple tool-based pick, transfer, and place task, inspired from the peg and transfer task used to train hand-eye coordination and bi-manual dexterity skills in laparoscopic surgery [11]. In the designed task, the user had to pick six small virtual cubes from a starting position using one tool, transfer them

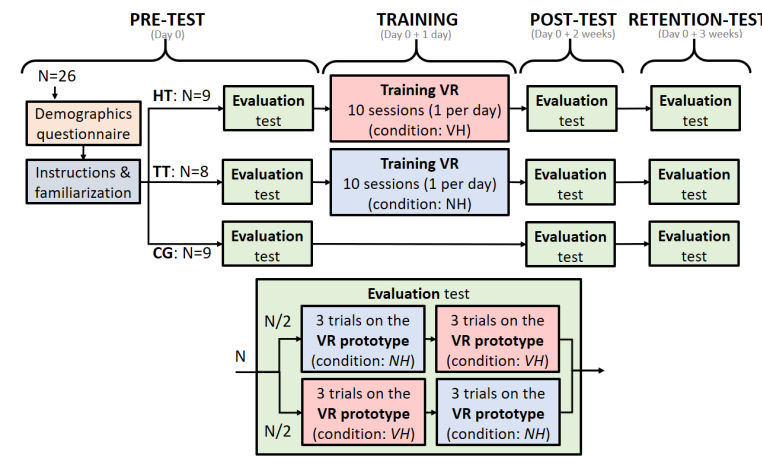

Figure 1: Experimental protocol for the longitudinal study

to another tool, and place them in a final square target. The start and target positions of each cube were fixed and numbered, and participants were required to place them in an ascendant order (from cube \#1 to cube \#6).

In addition, each cube had a green pattern on one of its faces. At the beginning, the cubes were oriented with the pattern facing upwards, and participants were required to place them with the pattern facing downwards, while aligning the cube with the target square. This feature made the task more challenging by forcing the participants to rotate the cubes during their transfer.

In addition, the working area was divided into two sub-spaces separated by a wall to constraint participants' use of tools. Indeed, each tool was used to interact with the cubes only in one sub-space, with the exception of the exchange zone (located on top of the virtual wall) where both tools could be used at the same time to transfer the cube from one side to the other. The task was also symmetrical: three cubes and three target squares were located at each side of the wall, and the participants were required to place each cube on the opposite sub-space, all while respecting the zone boundaries attributed to each tool (see Fig. 2).

To ensure that all the participants started from the same position for all the trials, they were required to place the tools in the starting position and wait for the countdown before each trial. They were asked to perform the experimental task as quickly and precisely as possible, and they were able to do as many cube manipulations using the tools as necessary. If a cube was dropped during the task, a dropping error counter was incremented but they were allowed to pick the cube up and continue with the task. Depending on which sub-space of the working area the cube was dropped in, they were instructed to use the appropriate tool (left or right) to pick it up. They were required to reduce the cube drops.

\subsection{Apparatus}

The VR prototype consisted of an $\mathrm{HTC} \mathrm{VIVE}^{1} \mathrm{HMD}$ to render an immersive experience from a first-person perspective, two haptic devices to control the virtual tools and to provide the force feedback output, and a pair of data gloves for tracking the users' hand and fingers movements (see Fig. 3).

Virtual environment: The VE consisted of a working area divided into two sub-spaces by a rigid wall. Three black square targets were designed in different positions and rotations on each sub-space. They were numbered from 1 to 3 in the left sub-space, and from 4 to 6 in the right one. The initial cube positions were also designed and numbered. Six pickable cubes with a green pattern on one of the faces were initially placed in the initial zones (cubes 1 to 3 on the right side, and 4 to 6 on the left side). In addition, two

\footnotetext{
${ }^{1}$ https://www.vive.com
} 

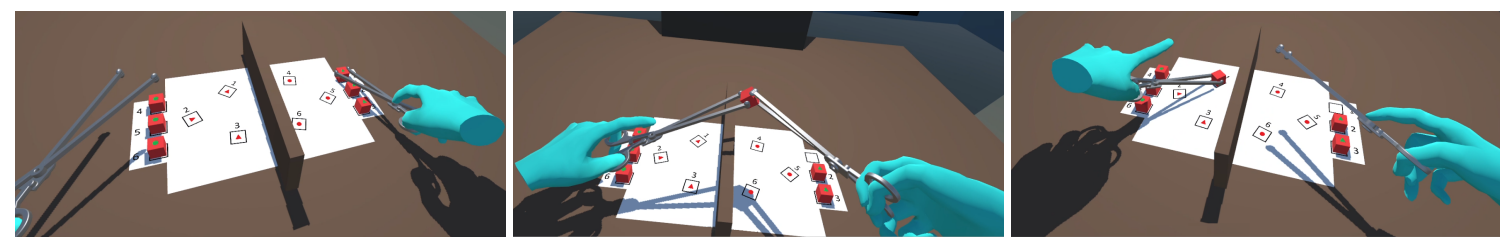

(a) Example of the task execution for cubes \#1 to \#3 (VH condition)

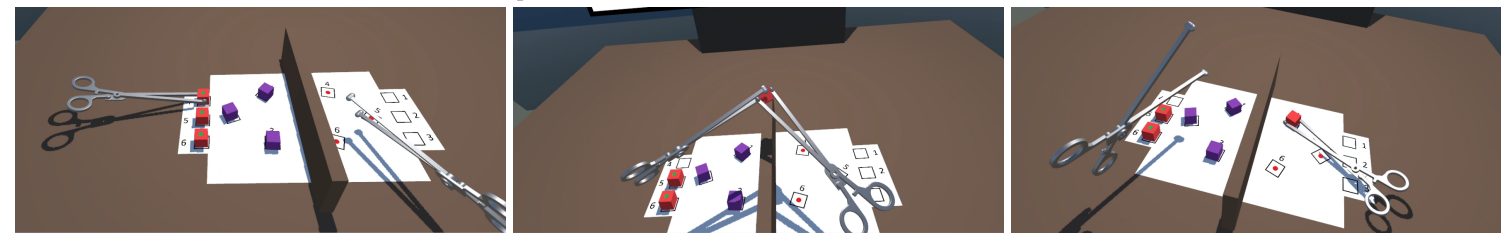

(b) Example of the task execution for cubes \#4 to \#6 (NH condition)

Figure 2: Pick, transfer and place task execution and experimental conditions

virtual forceps representing the tools and a pair of virtual hands were also part of the VE.

Physical interfaces: Two Geomagic Touch ${ }^{2}$ haptic devices (3D Systems Inc.) were placed in a wooden support. These interfaces provide $6 \mathrm{DOF}$ for movement and $3 \mathrm{DOF}$ for force feedback, and were used to control the virtual tools. We have replaced each stylus by a 3D printed forceps model, which were designed to include an extra DOF for opening and closing the tool. This allowed to simulate a realistic grabbing of the cubes. Indeed, a potentiometer was placed on the handles intersection axis of each tool to register the opening angle. Data was sent to the VE through an Arduino UNO board.

Hands representation: The Hi5 VR Gloves ${ }^{3}$ (Noitom International Inc., MA, USA) were used to track the users' hand and finger movements. These interfaces provide $1 \mathrm{DOF}$ for each finger, 1 additional DOF for the thumb, and 3 DOF for hand's rotation. The users' virtual hands global positions (3 DOF) were provided through an HTC tracker attached to each glove.

In terms of kinematic fidelity, we have provided $1 \mathrm{DOF}$ for flexion of each finger not directly involved in the tool grasping (free fingers, commanded by the data gloves sensors), and an extra DOF for simulating the "pinch" movement carried out with the (other) two main fingers to open and close the tool (controlled by the potentiometer). We have proposed three different configurations for the main fingers to contemplate participants comfort and tool-handling preference: the thumb and index, the thumb and middle, and the thumb and ring fingers (see Fig. 4). No spreading movements between the fingers were provided (limitations of the data gloves interface).

Finally, a moderate level of visual fidelity was used. Indeed, we have used a human-like hand model. However, a neutral skin color was used to symbolize the use of gloves. This made the final version look more cartoonish than realistic. In addition, neither the cosmetic aspects (e.g., skin texture), nor the dimensions and gender of the users' hands were personalized in our model.

Software implementation: The VR simulator was a clientserver application running on two different computers (CPU: Intel i7, GPU: GeForce GTX 1060/1070, RAM: 16 GB) directly connected through a UTP cable.

A C ++ server was implemented using the chai $3 \mathrm{~d}^{4}$ framework (version 3.2.0) [13] for haptic force feedback and ODE physics engine for collisions detection. It receives the physical interface positions and the potentiometer angles data, and computes the tools and virtual cubes interactions. In addition, it renders the collision

${ }^{2}$ https://www.3dsystems.com

${ }^{3}$ https://hi5vrglove.com

${ }^{4}$ https://www.chai3d.org forces when the tools are in contact with the virtual objects (cubes, wall, table surface), similar to real objects manipulation. Finally, it continuously transmits the tools and cubes positions and orientations to the client application (UDP socket).

The client application was developed using Unity $3 \mathrm{D}^{5}$ (version 2018.3.6) with C\#. It receives the computed positions and orientation of all the VE components and displays them in the HMD. Finally, it uses the gloves information to animate the virtual hands in real time.

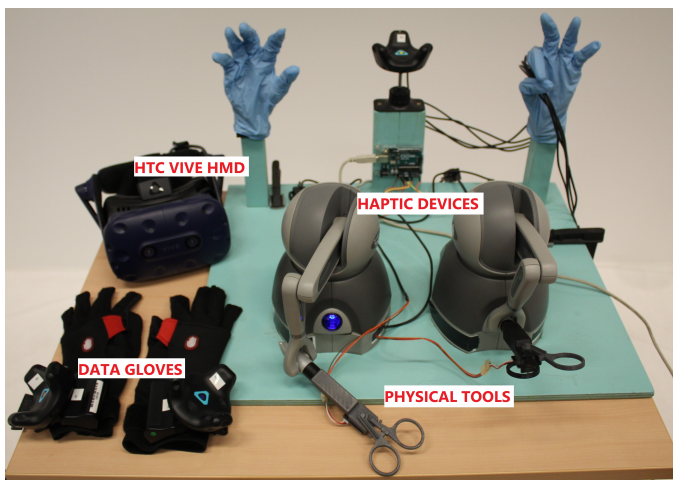

Figure 3: Interaction devices

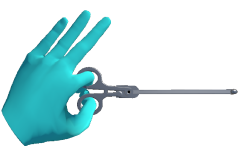

(a) Thumb and index

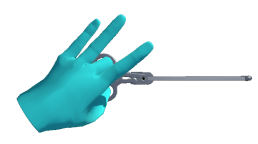

(b) Thumb and middle

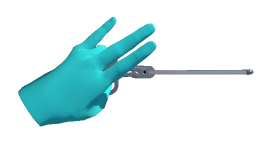

(c) Thumb and ring
Figure 4: The different configurations for hand-tool association

\subsection{Experimental procedure}

All participants $(N=26)$ started with the PRE phase (day 0$)$. The first step consisted of reading and signing the consent form, as well as completing the demographics questionnaire. Then, they were asked to read the experimental instructions presenting the VR prototype and the task to perform. Instructions on how to use the different devices was also provided. After that, the participants were asked to sit comfortably, put in the data gloves and wear the HMD. For the VH condition, the standard data gloves calibration was performed.

${ }^{5}$ https://unity.com 
To allow participants feeling comfortable with the setup and the experimental task, a familiarization session was first carried out. During this session, participants had to perform a pick, transfer and place of one cube on each side of the wall. Finally, they performed the experimental session, consisting of three trials of the experimental task on each condition ( $\mathrm{VH}$ and $\mathrm{NH}$ ). This procedure was repeated in the POST (day $0+2$ weeks) and RET (day $0+3$ weeks) phases for all the participants.

In addition, the training groups (HT and TT, $N=17$ ) participated in the TRAIN phase, which consisted of 30-minute training sessions in ten days during two weeks. The first training session took place the day after the PRE phase (day $0+1$ day), and the last one the day before the POST phase. For each training session, participants were installed in the VR prototype area, asked to sit comfortably and wear the gloves and the HMD. For participants in the HT group, a calibration of the data gloves was performed for each session. The participants were then asked to perform the experimental task ten times or the maximum number of trials possible in 20 minutes. They were allowed to rest their arms and remove the HMD between each trial if they felt tired or uncomfortable.

\subsection{Data collection and analyses}

The users' performance was evaluated through the task completion time, the accuracy of placing the cubes, and the number of cubes dropped during the task (error measure). Data was automatically recorded by the application on a log file.

The task completion time was calculated from the first contact of the tool with the first cube (cube \#1), until the placement of the last cube (cube \#6). The placement accuracy was based on the distance and the rotation difference between the cube and the target. The distance was calculated from the center of each cube's face in contact with the working area and the center of the corresponding square target. The rotation difference was the minimal angle difference between the cube's face in contact with the working area and the corresponding target square. The mean rotation difference and distance for the six cubes was retained as accuracy measure. Finally, an error was counted for cube drop.

\subsubsection{Data analyses}

All data analyses were performed using SPSS software version 21.0 (IBM Corp., Armonk, NY, USA) with the appropriate statistical tests. We have used a confidence level of $95 \%$ for all our statistical analyses. Therefore, a result is considered significant when $p<.05$.

The first step of the data analysis aimed to determine whether performance in the VR prototype was different between groups through time for the different conditions. We have used a three-way mixeddesign ANOVA on the pre-, post- and retention-test participants performance on the VR prototype (with two test conditions - VH and $\mathrm{NH}$ ), Tukey's HSD post hoc test, as well as pairwise comparisons with Bonferroni correction. Outliers were removed from the positional data and replaced with the group means before analysis. Two data points on the total time, three on the rotation difference and total drops, and four on the position distance were considered as outliers. Furthermore, residuals normal distribution for each cell of the study was validated through visual inspection of Q-Q plots, and homogeneity of variances through Levene's test. For the two- and three-way interaction effects, Mauchly's test of sphericity was calculated, and Huynh-Feldt correction applied when sphericity was not assumed ( $\varepsilon$ is reported). In addition, one-way ANOVAs were performed for the PRE phase measurements to compare groups initial performance (baseline) for both conditions. This aimed to determine whether all groups had a comparable performance at the beginning of the experiment. Moreover, to assess the training impact, independent-samples t-tests were applied to compare groups performance between PRE and POST phases for each condition separately, and paired-samples t-tests were employed to compare, for each group, the participants' performance between the two conditions.

\section{Results}

Descriptive statistics are summarized in Table 1.

\subsection{Baseline}

One-way ANOVAs indicate no significant effects $(p>.05)$ for all the dependent measures, indicating that all the participants had a similar performance level at the beginning of the experiment.

\subsection{Performance}

The results of the three-way mixed-design ANOVA for each objective measure are summarized in Table 2 and Fig. 5. Only significant results for each measure are reported below.

Time: For the total time, no significant three-way interaction effect between the phase, the condition and the group was found. In addition, no significant two-way interaction effects were found neither between phase and condition was found, nor between group and condition. A significant two-way interaction effect between phase and group $(F(2.5,28.2)=5.28, p=.008$, $\left.\eta^{2}=.314, \varepsilon=.614\right)$. Finally, a significant main effect of phase $\left(F(1.2,28.2)=86.76, p<.001, \eta^{2}=.790, \varepsilon=.614\right)$ and group $\left(F(2,23)=6.38, p=.006, \eta^{2}=.357\right)$ were found. No significant main effect of the condition was observed. Tuckey's HSD post hoc test showed a significant difference between control group and both, hand-training $(p=.024)$ and tool-training groups $(p=.009)$. Pairwise comparisons with Bonferroni correction showed that total time at PRE was statistically significantly different from total time at POST and RET phases $(p<.001)$, and POST was statistically significantly different from RET phase $(p=.011)$.

Accuracy: No significant three-way interaction effect was found for any of the accuracy measures. In addition, there were no significant two-way interaction effects neither between phase and condition, nor between group and condition for these measures. There was a significant two-way interaction effect of group and phase for both, the position distance $(F(4,46)=5.60, p=.001$, $\left.\eta^{2}=.328\right)$, and the rotation difference $(F(4,46)=4.16, p=.008$, $\left.\eta^{2}=.266\right)$ accuracy measures. A significant main effect of phase was also found for both measures $(F(2,46)=32.66, p<.001$, $\eta^{2}=.587$, and $F(2,46)=18.26, p<.001, \eta^{2}=.443$; respectively for position and rotation). No significant main effect of group was found for these measures, and a significant main effect of the condition was found only for the position distance measure $\left(F(2,23)=6.62, p=.017, \eta^{2}=.223\right)$. Pairwise comparisons with Bonferroni correction showed that both accuracy measures performance at PRE were statistically significantly different from those at POST phase $(p<.001)$, suggesting learning, and no significant difference was found for these measures between POST and RET phases ( $p<.746, p=1.00$; respectively for position and rotation), suggesting no skill decay.

Errors: No significant three-way interaction effect was found for the cubes dropped. Moreover, there were no significant twoway interaction effects between phase and condition, nor between group and condition. A significant two-way interaction effect between group and phase was observed $(F(2.9,33.2)=3.42, p=.030$, $\left.\eta^{2}=.229\right)$. There was a significant main effect of the phase $\left(F(1.4,33.2)=22.90, p<.001, \eta^{2}=.499\right)$. No significant main effect of the condition was found, neither a group main effect. Pairwise comparisons with Bonferroni correction showed that PRE and POST were statistically significantly different for the total cubes dropped $(p<.001)$, suggesting learning, and no significant difference was found between POST and RET error $(p=.900)$, indicating no skill decay one week after the training period. 
Table 1: Mean and standard deviation for dependent variables

\begin{tabular}{|c|c|c|c|c|c|c|}
\hline Phase & $\begin{array}{l}\text { Condi- } \\
\text { tion }\end{array}$ & Group & $\begin{array}{l}\text { Total time (s) } \\
(\mu(\sigma))\end{array}$ & $\begin{array}{l}\text { Position (mm) } \\
(\mu(\sigma))\end{array}$ & $\begin{array}{l}\text { Rotation }\left(^{\circ}\right) \\
(\mu(\sigma))\end{array}$ & $\begin{array}{l}\text { Drops (Qty.) } \\
(\mu(\sigma))\end{array}$ \\
\hline \multirow{2}{*}{ PRE-TEST } & VH & $\begin{array}{l}\text { CG } \\
\text { HT } \\
\text { TT }\end{array}$ & $\begin{array}{l}142.65(41.13) \\
138.45(42.84) \\
120.88(19.37) \\
\end{array}$ & $\begin{array}{l}3.99(1.04) \\
4.35(1.77) \\
4.07(0.78) \\
\end{array}$ & $\begin{array}{l}9.99(5.80) \\
12.17(5.98) \\
9.74(3.85)\end{array}$ & $\begin{array}{l}5.63(3.12) \\
7.04(7.00) \\
5.50(2.44) \\
\end{array}$ \\
\hline & NH & $\begin{array}{l}\text { CG } \\
\text { HT } \\
\text { TT }\end{array}$ & $\begin{array}{l}122.68(14.42) \\
142.08(14.42) \\
144.56(44.04)\end{array}$ & $\begin{array}{l}4.39(2.34) \\
4.51(2.12) \\
4.42(1.19)\end{array}$ & $\begin{array}{l}9.40(4.79) \\
12.56(8.48) \\
10.28(5.16)\end{array}$ & $\begin{array}{l}5.22(4.43) \\
6.59(5.55) \\
5.95(2.29)\end{array}$ \\
\hline \multirow{2}{*}{ POST-TEST } & VH & $\begin{array}{l}\text { CG } \\
\text { HT } \\
\text { TT }\end{array}$ & $\begin{array}{l}104.41(9.02) \\
74.82(15.10) \\
72.28(12.35)\end{array}$ & $\begin{array}{l}3.78(1.28) \\
1.88(0.80) \\
1.86(0.60) \\
\end{array}$ & $\begin{array}{l}8.74(3.20) \\
3.26(1.00) \\
5.58(3.34) \\
\end{array}$ & $\begin{array}{l}4.12(2.22) \\
1.11(1.21) \\
1.42(1.32) \\
\end{array}$ \\
\hline & NH & $\begin{array}{l}\text { CG } \\
\text { HT } \\
\text { TT }\end{array}$ & $\begin{array}{l}115.93(22.69) \\
75.43(11.45) \\
76.20(11.17)\end{array}$ & $\begin{array}{l}4.59(1.36) \\
2.54(1.24) \\
2.56(0.98)\end{array}$ & $\begin{array}{l}9.61(4.91) \\
3.65(1.20) \\
5.15(2.37)\end{array}$ & $\begin{array}{l}5.19(3.47) \\
1.24(2.07) \\
1.23(0.56)\end{array}$ \\
\hline \multirow{2}{*}{ RETENTION-TEST } & VH & $\begin{array}{l}\text { CG } \\
\text { HT } \\
\text { TT }\end{array}$ & $\begin{array}{l}96.95(16.40) \\
73.29(10.79) \\
69.25(12.57)\end{array}$ & $\begin{array}{l}3.17(0.91) \\
2.12(1.19) \\
2.52(1.68)\end{array}$ & $\begin{array}{l}7.50(6.00) \\
4.83(5.18) \\
6.03(4.31) \\
\end{array}$ & $\begin{array}{l}4.41(3.62) \\
0.93(1.33) \\
1.13(0.85) \\
\end{array}$ \\
\hline & $\mathrm{NH}$ & $\begin{array}{l}\text { CG } \\
\text { HT } \\
\text { TT }\end{array}$ & $\begin{array}{l}106.53(12.84) \\
70.78(7.96) \\
71.31(11.62)\end{array}$ & $\begin{array}{l}3.06(1.09) \\
2.23(0.95) \\
2.69(1.64)\end{array}$ & $\begin{array}{l}8.15(5.40) \\
3.52(1.43) \\
5.73(3.17)\end{array}$ & $\begin{array}{l}3.83(2.05) \\
0.52(0.63) \\
1.44(1.31)\end{array}$ \\
\hline
\end{tabular}

Table 2: Results of the two-way mixed design ANOVAs for each dependent variable.

\begin{tabular}{|c|c|c|c|}
\hline Measure & Phase $\times$ Condition $\times$ Group & Phase $\times$ Condition & $\varepsilon^{\diamond}$ \\
\hline $\begin{array}{l}\text { Total time } \\
\text { Position } \\
\text { Rotation } \\
\text { Drops }\end{array}$ & $\begin{array}{l}F(2.7,31.2)=3.01, p=.050, \eta^{2}=.207 \\
F(3.7,42.1)=0.14, p=.960, \eta^{2}=.012 \\
F(4,46)=0.73, p=.576, \eta^{2}=.060 \\
F(4,46)=0.30, p=.879, \eta^{2}=.025\end{array}$ & $\begin{array}{l}F(1.4,31.2)=0.93, p=.836 \eta^{2}=.004 \\
F(1.8,42.1)=2.41, p=.106, \eta^{2}=.091 \\
F(2,46)=0.26, p=.773, \eta^{2}=.011 \\
F(2,46)=0.21, p=.808, \eta^{2}=.009\end{array}$ & $\begin{array}{l}.678 \\
.914\end{array}$ \\
\hline Measure & Group $\times$ Phase & Phase & $\varepsilon^{\diamond}$ \\
\hline $\begin{array}{l}\text { Total time } \\
\text { Position } \\
\text { Rotation } \\
\text { Drops }\end{array}$ & $\begin{array}{l}F(2.5,28.2)=5.28, p=.008 *, \eta^{2}=.314 \\
F(4,46)=5.60, p=.001 *, \eta^{2}=.328 \\
F(4,46)=4.16, p=.008 *, \eta^{2}=.266 \\
F(2.9,33.2)=3.42, p=.030 *, \eta^{2}=.229\end{array}$ & $\begin{array}{l}F(1.2,28.2)=86.76, p<.001 *, \eta^{2}=.790 \\
F(2,46)=32.66, p<.001 *, \eta^{2}=.587 \\
F(2,46)=18.26, p<.001 *, \eta^{2}=.443 \\
F(1.4,33.2)=22.90, p<.001 *, \eta^{2}=.499\end{array}$ & .721 \\
\hline Measure & Condition $\times$ Group & Condition & \\
\hline $\begin{array}{l}\text { Total time } \\
\text { Position } \\
\text { Rotation } \\
\text { Drops }\end{array}$ & $\begin{array}{l}F(2,23)=0.81, p=.458, \eta^{2}=.066 \\
F(2,23)=0.36, p=.965, \eta^{2}=.003 \\
F(2,23)=0.16, p=.851, \eta^{2}=.014 \\
F(2,23)=0.20, p=.823, \eta^{2}=.017\end{array}$ & $\begin{array}{l}F(2,23)=1.11, p=.302, \eta^{2}=.046 \\
F(2,23)=6.62, p=.017 *, \eta^{2}=.223 \\
F(2,23)<0.01, p=.952, \eta^{2}<.001 \\
F(2,23)<0.01, p=.979, \eta^{2}<.001\end{array}$ & \\
\hline Measure & Group & & \\
\hline $\begin{array}{l}\text { Total time } \\
\text { Position } \\
\text { Rotation } \\
\text { Drops }\end{array}$ & $\begin{array}{l}F(2,23)=6.38, p=.006 *, \eta^{2}=.357 \\
F(2,23)=1.98, p=.162, \eta^{2}=.147 \\
F(2,23)=1.04, p=.369, \eta^{2}=.083 \\
F(2,23)=2.90, p=.075, \eta^{2}=.201\end{array}$ & & \\
\hline
\end{tabular}

To summarize, the results indicate that participants improved their task completion time as an effect of the training phase, with the training groups achieving a better performance than the control group. We can also observe that participants in the control group have a higher and more accelerated improvement for time when evaluated in the VH condition. It can also be observed that participants, in general, continue to learn from post- to retention-test, suggesting that total time can still be improved. Participants accuracy was mea- sured as the distance error and angle difference between the placed cube and the target square. For both measures, we can observe that participants improved between pre- and post-test, suggesting a learning effect, while keeping almost the same performance between post- and retention-test, indicating that they were able to retain the learned skills after training stopped. Finally, the same results can be observed for the total cubes dropped, where participants improved significantly from pre- to post-test, and maintained their performance 


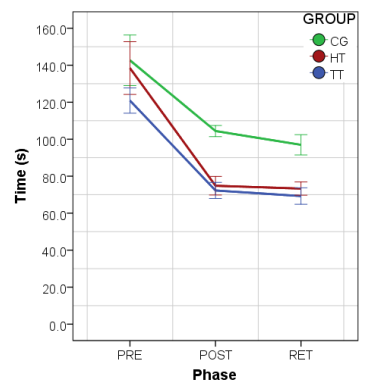

(a) Total time (VH)

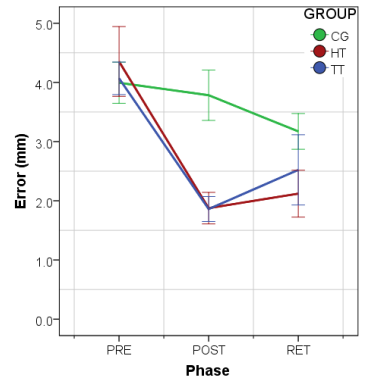

(c) Position distance (VH)

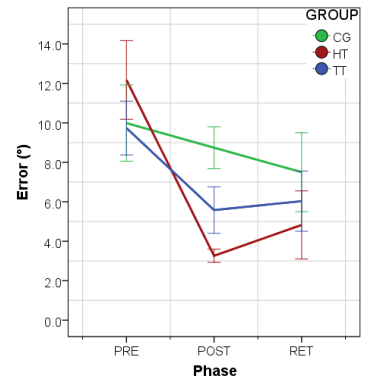

(e) Rotation difference (VH)

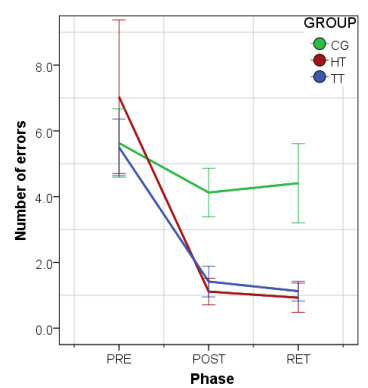

(g) Total drops (VH)

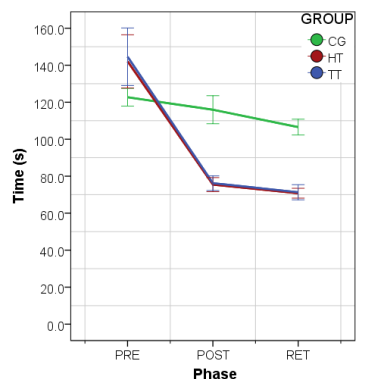

(b) Total time (NH)

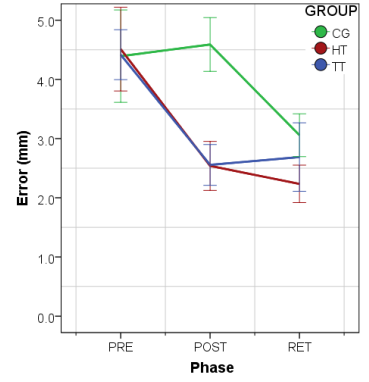

(d) Position distance $(\mathrm{NH})$

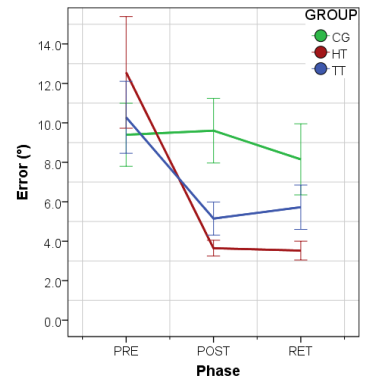

(f) Rotation difference (NH)

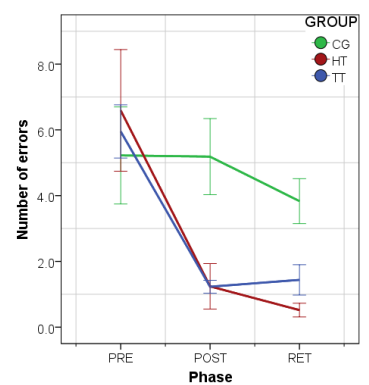

(h) Total collisions (NH)
Figure 5: Performance measures

in the retention-test. As a whole, these results suggest that training in VR (both groups) improved the participants' performance as compared to the control group.

\subsection{Learning}

The change in performance from PRE to POST as an indicator of learning was analyzed for each experimental condition separately by comparing each group 2-by-2. In addition, for each group, we have compared this change in performance between the two conditions.

VH condition: Results from individual unpaired t-tests showed that the change in performance for the hand-training group compared to the control group was significantly different for the position distance $(t=-3.05, p=.008)$, and the rotation difference $(t=-3.08, p=.007)$. The change in performance for the tool-training group compared to the control group was significantly different for the position distance measure $(t=-3.41, p=.004)$. No other differences were found.

NH condition: Results from individual unpaired t-tests showed that the change in performance for the hand-training group compared to the control group was significantly different for the total time $(t=-4.00, p=.001)$, the position distance $(t=-2.93$, $p=.010)$, the rotation difference $(t=-3.44, p=.007)$, and the total drops $(t=-2.16, p=.046)$ measures. The change in performance for the tool-training group compared to the control group was significantly different for the total time $(t=-3.53, p=.003)$, the position distance $(t=-3.33, p=.007)$, the rotation difference $(t=-2.74, p=.015)$, and the total drops $(t=-2.25, p=.040)$ measures. No differences were found between the training groups.

VH vs. NH condition: Results from individual pairedsamples t-tests showed that the change in performance between the two conditions were not significantly different for any of the four objective measures for the hand-training group, neither for the tool-training group, nor for the control group.

To summarize, for the VR prototype with the VH condition (virtual hand visualization), participants that trained under that condition (HT) significantly improved their accuracy as compared to the $\mathrm{CG}$, but not the total time and the errors made, although having better results. This can be explained by the fact that the $\mathrm{VH}$ condition allowed participants in the CG to significantly improve their time in only two sessions. Participants in the other training group (TT), who trained without the virtual hand visualization, also improved their accuracy in the VR prototype with the VH condition as compared to the CG, suggesting transfer from the $\mathrm{NH}$ to the $\mathrm{VH}$ condition. Both training groups significantly improved their performance (for all measures) in the VR prototype with the $\mathrm{NH}$ condition, when compared to the CG. This also shows transfer from the $\mathrm{VH}$ to the $\mathrm{NH}$ condition. Finally, the improvement from pre- to post-test was not different between the two conditions nor for the training groups, neither for the control group. This suggests that the performance improvement in the VR prototype is not affected by the experimental condition (virtual hand visualization vs. tools only visualization).

\section{Discussion}

\subsection{VR prototype for learning}

The results of this longitudinal study show that participants improved their task completion time, accuracy (the distance error and angle difference between the placed cube and the target square), and errors (the total cubes dropped) as an effect of the training phase, with the training groups achieving a better whole performance than the control group. This implies learning in the VR prototype, and allows us to validate $\mathrm{H} 1$. Indeed, the training groups reduced between $40 \%$ to $48 \%$ the general mean task completion time between pre- and post-test, between $42 \%$ to $56 \%$ the distance accuracy on placement, between $42 \%$ to $73 \%$ the rotation difference, and between $74 \%$ to $84 \%$ the total number of cubes dropped. Whereas the control group reported to have lower improvements than the training groups, less than 5\% improvement of performance between pre- and post-test for all the measures in the VR prototype under the no hand condition, and between $5 \%$ and $27 \%$ for the virtual hand condition. In addition, no differences were reported between the hand-training and tooltraining as a function of group.

Moreover, for both accuracy measures (rotation and position) and the total number of errors, the results also show that participants kept almost the same performance between post- and retention-test, indicating that they were able to retain the learned skills after a short break in training. To fully assess retention of motor skills, a longer break should be given between training and retention-test, since 
motor skills do not decay in a short period of time. These results reinforce the learning effectiveness of the VR simulator for these performance measures. However, the task completion time was significantly different between the post- and retention-test, which means that participants in general continue to improve their speed after training. They reduced $2 \%$ to $8 \%$ the total task completion time between these phases (post and retention), suggesting that there is still margin for improvement in terms of the total time to perform the task before reaching a plateau.

As a whole, these results suggests that the training of the pick and place task in our VR simulator (both groups) improves the participants' performance as compared to the control group (H1 validated for all the performance measures).

\subsection{Hand visualization training condition}

The results show that for the VR prototype with the virtual hand condition, participants that trained under that condition (hand-training group) significantly improved their accuracy as compared to the control group, but not the total time and the errors made, although having better results than participants in that group. One explanation for this can be that the VR prototype with the virtual hand condition allowed the control group participants to significantly improve their task completion time in only two sessions. Indeed, participants in the control group had a higher and more accelerated improvement for time when evaluated in the virtual hand condition, $25 \%$ time reduction for virtual hand condition compared to $6 \%$ for the no hand condition. Participants in the other training group (tool-training), which trained without the virtual hand representation also improved their accuracy in the VR prototype with the virtual hand condition as compared to the control group, suggesting transfer of learning for accuracy from the no hand to the virtual hand condition.

Moreover, both training groups significantly improved their performance (for all measures) in the VR prototype with the no hand condition, when compared to the control group. This also shows a learning transfer in the other direction, from the virtual hand training condition to the no hand condition, for all the performance measures.

On the other hand, the results also show that the participants in the hand-training group were more accurate than those in the tool-training group in aligning the cubes during placement (rotation difference accuracy), with a higher improvement (between pre- and post-test) of $70 \%$ and $73 \%$ for the hand-training group, compared to $43 \%$ and $49 \%$ for the tool-training group, respectively for the evaluation in the VR prototype under the virtual hand and no hand conditions, although no main effect of the condition was found in the three-way ANOVA for this measure. The lack of significant value can be related to the small number of participants, generating a small effect size. Indeed, the calculated sample size for training might not be enough to visualize the effect of hand visualization on training. Further studies are required to determine the real impact of hand representation on accuracy for tool-based tasks training.

Additionally, when considering the comparison of learning (pre minus post) between the two conditions analyzed separately for each individual group, the improvement was not different between the two conditions, nor for the training groups, neither for the control group. This suggests that the performance improvement in the VR prototype is not affected by the training condition (virtual hands visualization vs. tools only visualization). One possible explanation might be the fact that participants were more concentrated in the task itself than the visual representation of the tools and hands. This does not allow us to validate our second hypothesis $(\mathrm{H} 2)$.

As a whole, we do not observe a significant impact of users' hand representation and control on users' training for tool-based motor skills. Indeed, only small (not significant) improvements concerning the rotation difference accuracy measure in favor of having the hands representation were found. Nonetheless, only a partial embodiment was tested, and for a task not demanding much of finger dexterity.
As mentioned before, participants could have been more focused on the cubes, and included the tools as extensions of their own hands. Therefore, further experiments are needed to investigate the effect of hand representation on other tasks.

\section{Conclusion}

In the present work, we have explored how the users' hand visualization affects the training of a motor task in an immersive virtual reality simulator. Our main objective was to investigate the impact of visualizing virtual hands on participants' task performance after a two weeks training period in a tool-based motor skills simulator. In order to answer this question, we have created a VR prototype for a tool-based pick and place task inspired from existing surgical simulators. This prototype permitted to visualize the users' hands in the VE through a human-like hands model collocated with the users' real hands, and which reproduced also their finger movements.

In order to evaluate participants' performance outcome after training, we have designed a pre-, post- and retention-test user experiment with two training groups (one trained under the visualization of the virtual hands condition, and the other without it) and a control group which received no training.

The results of this study shows that users improved their performance after the two-week training period in the VR simulator for both training groups when compared to the control group. The results show also short-term skills retention after training has stopped. This indicates that our simulator is an efficient trainer for the designed task. On the other hand, there was no performance difference between the two training groups after training. However, the small sample size of participants considered might have limited the visualization of a significant effect.

In general, these results add to the existing literature on motor skills trainers design, providing further insights into the effects of partial embodiment by adding evidence that for tool-based motor tasks, hand visualization might not directly impact performance. These findings suggest that tool-based motor skills trainers in general can be simplified, by including less cumbersome equipment (i.e., data gloves, device communication devices, etc.) that can further increase their costs.

\section{LIMITATIONS AND FUTURE WORKS}

The main limitation of the study is the number of participants included in the study, therefore, results should be considered with caution regarding power analysis.

Another limitation of our user study is that only one type of hands representation was evaluated in terms of fidelity classification. Indeed, previous studies have been shown that performance can be impacted by the kinematic fidelity of the hand representation [25], that a realistic personalized hand can improve object size estimation [22], and that object-size perception in VR is affected by antropomorphic hands' size [27]. Further studies with different levels of visual appearance and kinematic properties are needed to increase our knowledge on this topic and to be able to generalize these results, which suggest that partial-embodiment generated by virtual hands does not impact tool-based motor training when compared to providing only the tools visualization.

In the future, we plan to include a real-world training and test condition, to compare VR training to traditional approaches. This could add knowledge concerning the effectiveness of immersive VR for training and transfer (external validity) of knowledge to the real environment. To do so, a physical prototype recreating the simulated environment is required to analyze users' performance on the real task. This will further validate the use of immersive VR simulators for training motor skills tasks for surgery and other application domains. 


\section{REFERENCES}

[1] F. Argelaguet, L. Hoyet, M. Trico, and A. Lécuyer. The role of interaction in virtual embodiment: Effects of the virtual hand representation. In 2016 IEEE Conference on Virtual Reality (VR), pp. 3-10. IEEE, 2016. doi: 10.1109/VR.2016.7504682

[2] V. S. Arikatla, G. Sankaranarayanan, W. Ahn, A. Chellali, S. De, C. G. L. Cao, J. Hwabejire, M. DeMoya, S. Schwaitzberg, and D. B. Jones. Face and construct validation of a virtual peg transfer simulator. Surgical endoscopy, 27(5):1721-1729, 2013. doi: 10.1007/s00464-012 $-2664-y$

[3] J. Balcombe. Medical training using simulation: toward fewer animals and safer patients. Alternatives to Laboratory Animals, 32(1_suppl):553-560, 2004. doi: 10.1177/026119290403201s90

[4] A. U. Batmaz, M. de Mathelin, and B. Dresp-Langley. Getting nowhere fast: trade-off between speed and precision in training to execute imageguided hand-tool movements. BMC psychology, 4(1):1-19, 2016.

[5] A. U. Batmaz, M. de Mathelin, and B. Dresp-Langley. Seeing virtual while acting real: Visual display and strategy effects on the time and precision of eye-hand coordination. PloS one, 12(8):e0183789, 2017.

[6] B. S. Bloom. Automaticity:" the hands and feet of genius.". Educational leadership, 43(5):70-77, 1986.

[7] P. Bradley. The history of simulation in medical education and possible future directions. Medical Education, 40(3):254-262, 2006. doi: 10 1111/j.1365-2929.2006.02394.x

[8] C. Buckley, E. Nugent, D. Ryan, and P. Neary. Virtual reality - a new era in surgical training. In C. Eichenberg, ed., Virtual reality in psychological, medical and pedagogical applications, chap. 7, pp. 139-166. InTech, 2012. doi: 10.5772/46415

[9] G. Caggianese, L. Gallo, and P. Neroni. The vive controllers vs. leap motion for interactions in virtual environments: a comparative evaluation. In International Conference on Intelligent Interactive Multimedia Systems and Services, pp. 24-33. Springer, 2018.

[10] J. C. Castro-Alonso, P. Ayres, and F. Paas. Dynamic visualisations and motor skills. In W. Huang, ed., Handbook of human centric visualization, chap. 6, pp. 551-580. Springer, 2014. doi: 10.1007/978 -1-4614-7485-2_22

[11] A. Chellali, H. Mentis, A. Miller, W. Ahn, V. S. Arikatla, G. Sankaranarayanan, D. Suvranu, S. D. Schwaitzberg, and C. G. L. Cao. Achieving interface and environment fidelity in the Virtual Basic Laparoscopic Surgical Trainer. International Journal of Human-Computer Studies, 96:22-37, 2016. doi: 10.1016/j.ijhcs.2016.07.005

[12] T. R. Coles, D. Meglan, and N. W. John. The role of haptics in medical training simulators: a survey of the state of the art. IEEE Transactions on Haptics, 4(1):51-66, 2011. doi: 10.1109/TOH.2010.19

[13] F. Conti, F. Barbagli, R. Balaniuk, M. Halg, C. Lu, D. Morris, L. Sentis, J. Warren, O. Khatib, and K. Salisbury. The chai libraries. In Proceedings of Eurohaptics 2003, pp. 496-500, 2003.

[14] C. G. Corrêa, F. L. Nunes, E. Ranzini, R. Nakamura, and R. Tori. Haptic interaction for needle insertion training in medical applications: The state-of-the-art. Medical Engineering \& Physics, 63:6-25, 2018. doi: 10.1016/j.medengphy.2018.11.002

[15] D. Escobar-Castillejos, J. Noguez, L. Neri, A. Magana, and B. Benes. A Review of Simulators with Haptic Devices for Medical Training. Journal of Medical Systems, 40(4):104-126, 2016. doi: 10.1007/s10916 $-016-0459-8$

[16] L. Freina and M. Ott. A literature review on immersive virtual reality in education: state of the art and perspectives. In The international scientific conference elearning and software for education, vol. 1, pp. 10-1007, 2015

[17] M. González-Franco, T. C. Peck, A. Rodríguez-Fornells, and M. Slater A threat to a virtual hand elicits motor cortex activation. Experimental Brain Research, 232(3):875-887, 2014. doi: 10.1007/s00221-013-3800 $-1$

[18] M. Guerraz, A. Breen, L. Pollidoro, M. Luyat, and A. Kavounoudias. Contribution of visual motion cues from a held tool to kinesthesia. Neuroscience, 388:11-22, 2018. doi: 10.1016/j.neuroscience.2018.06. 048

[19] T. Gutierrez, J. Rodriguez, Y. Velaz, S. Casado, A. Suescun, and E. J. Sanchez. Ima-vr: a multimodal virtual training system for skills transfer in industrial maintenance and assembly tasks. In 19th International Symposium in Robot and Human Interactive Communication, pp. 428433. IEEE, 2010.

[20] G. Henshall, S. R. Pop, M. R. Edwards, L. ap Cenydd, and N. W. John. Towards a high fidelity simulation of the kidney biopsy procedure. In 2015 IEEE Conference on Virtual Reality (VR), pp. 191-192. IEEE, 2015. doi: 10.1109/VR.2015.7223360

[21] W. A. IJsselsteijn, Y. A. W. de Kort, and A. Haans. Is this my hand i see before me? the rubber hand illusion in reality, virtual reality, and mixed reality. Presence: Teleoperators and Virtual Environments, 15(4):455-464, 2006.

[22] S. Jung, G. Bruder, P. J. Wisniewski, C. Sandor, and C. E. Hughes. Over my hand: Using a personalized hand in vr to improve object size estimation, body ownership, and presence. In Proceedings of the Symposium on Spatial User Interaction, SUI'18, pp. 60-68. ACM, 2018. doi: $10.1145 / 3267782.3267920$

[23] K. Kilteni, R. Groten, and M. Slater. The sense of embodiment in virtual reality. Presence: Teleoperators and Virtual Environments, 21(4):373-387, 2012. doi: 10.1162/PRES_a_00124

[24] A. M. Linsk, K. R. Monden, G. Sankaranarayanan, W. Ahn, D. B. Jones, S. De, S. D. Schwaitzberg, and C. G. Cao. Validation of the vblast pattern cutting task: a learning curve study. Surgical endoscopy, 32(4):1990-2002, 2018. doi: 10.1007/s00464-017-5895-0

[25] B. Lok, S. Naik, M. Whitton, and F. P. Brooks. Effects of handling real objects and self-avatar fidelity on cognitive task performance and sense of presence in virtual environments. Presence: Teleoperators and Virtual Environments, 12(6):615-628, 2003. doi: 10.1162/ 105474603322955914

[26] C. Lougiakis, A. Katifori, M. Roussou, and I.-P. Ioannidis. Effects of virtual hand representation on interaction and embodiment in hmdbased virtual environments using controllers. In 2020 IEEE Conference on Virtual Reality and 3D User Interfaces (VR), pp. 510-518. IEEE, 2020.

[27] N. Ogawa, T. Narumi, and M. Hirose. Virtual hand realism affects object size perception in body-based scaling. In 2019 IEEE Conference on Virtual Reality and 3D User Interfaces (VR), pp. 519-528. IEEE 2019. doi: 10.1109/VR.2019.8798040

[28] O. Ossmy and R. Mukamel. Short term motor-skill acquisition improves with size of self-controlled virtual hands. PloS one, 12(1), 2017. doi: 10.1371/journal.pone.0168520

[29] M. Poyade, L. Molina Tanco, A. Reyes Lecuona, A. Langley, E. Frutos, $\mathrm{S}$. Flores, et al. Validation of a haptic virtual reality simulation in the context of industrial maintenance. 2012.

[30] A. Ricca, A. Chellali, and S. Otmane. Influence of hand visualization on tool-based motor skills training in an immersive VR simulator. In IEEE International Symposium on Mixed and Augmented Reality (ISMAR), ISMAR 2020, pp. 1-8. IEEE, 2020.

[31] V. Schwind, P. Knierim, L. Chuang, and N. Henze. "where's pinky?" the effects of a reduced number of fingers in virtual reality. In Proceedings of the Annual Symposium on Computer-Human Interaction in Play, CHI PLAY '17, pp. 507-515. Association for Computing Machinery, 2017. doi: 10.1145/3116595.3116596

[32] V. Schwind, P. Knierim, C. Tasci, P. Franczak, N. Haas, and N. Henze. "these are not my hands!" effect of gender on the perception of avatar hands in virtual reality. In Proceedings of the 2017 CHI Conference on Human Factors in Computing Systems, CHI '17, pp. 1577-1582. Association for Computing Machinery, 2017. doi: 10.1145/3025453. 3025602

[33] R. Skarbez, F. P. Brooks, Jr, and M. C. Whitton. A survey of presence and related concepts. ACM Computing Surveys (CSUR), 50(6):1-39, 2017.

[34] M. Slater. Place illusion and plausibility can lead to realistic behaviour in immersive virtual environments. Philosophical Transactions of the Royal Society B: Biological Sciences, 364(1535):3549-3557, 2009.

[35] M. Slater, D. Pérez Marcos, H. Ehrsson, and M. V. Sanchez-Vives. Inducing illusory ownership of a virtual body. Frontiers in neuroscience, 3:29, 2009.

[36] H. G. Stassen, H. J. Bonjer, C. A. Grimbergen, and J. Dankelman. The future of minimally invasive surgery and training. In J. Dankelman, C. A. Grimbergen, and H. G. Stassen, eds., Engineering for patient 
safety: issues in minimally invasive procedures, chap. 12, pp. 272-282. CRC Press, 2005.

[37] R. Sung, J. M. Ritchie, T. Lim, R. Dewar, and N. Weston. Knowledge capture inside a haptic soldering environment. In Proceedings of the 6th International Conference on Virtual Learning, Workshop on Haptic Feedback Systems in Education, 2011.

[38] S. Ullrich and T. Kuhlen. Haptic Palpation for Medical Simulation in Virtual Environments. IEEE Transactions on Visualization and Computer Graphics, 18(4):617-625, 2012. doi: 10.1109/TVCG.2012. 46

[39] D. Van Nguyen, S. B. Lakhal, and A. Chellali. Preliminary evaluation of a virtual needle insertion training system. In 2015 IEEE Conference on Virtual Reality (VR), pp. 247-248. IEEE, 2015. doi: 10.1109/VR. 2015.7223388

[40] B. Wu, X. Yu, and X. Gu. Effectiveness of immersive virtual reality using head-mounted displays on learning performance: A meta-analysis. British Journal of Educational Technology, 51(6):1991-2005, 2020.

[41] L. Zhang, G. Sankaranarayanan, V. S. Arikatla, W. Ahn, C. Grosdemouge, J. M. Rideout, S. K. Epstein, S. De, S. D. Schwaitzberg, D. B. Jones, and C. G. Cao. Characterizing the learning curve of the vblast$\mathrm{pt}(\odot)$ (virtual basic laparoscopic skill trainer). Surgical endoscopy, 27(10):3603-3615, 2013. doi: 10.1007/s00464-013-2932-5 